\title{
Severe mitral valve prolapse with flail posterior leaflet and severe mitral regurgitation
}

\begin{abstract}
A 70-year-old female patient presented with shortness of breath, progressive dyspnea and chest discomfort for two weeks. A typical grade 3/6 pansystolic murmur was heard at the apex with radiation to the axillae. Both transthoracic and transesophageal echocardiography demonstrated an anteriorly directed eccentric jet of severe mitral regurgitation with mitral valve prolapse and flail posterior leaflet due to torn chordae. Localized P2 segment defect was identified by transesophageal echocardiography. The patient took diuretics and ACEI to improve her symptoms, followed by an operation of minimally invasive mitral valve repair. This case demonstrates that mitral valve prolapse with flail leaflet is a common cause of severe mitral regurgitation and echocardiography plays vital important role in identifying pathology of mitral valve prolapse with flail leaflet, assessment of mitral regurgitation severity and hemodynamic consequence, and planning mitral valve surgery.
\end{abstract}

Volume 7 Issue 4 - 2016

\section{Chaoran Yi}

Total Cardiology and Alberta Health Services, Canada

Correspondence: Chaoran Yi, Total Cardiology and Alberta Health Services, 4515 Varsity Dr NW, Calgary, AB T3A 0Z8, Canada,Tel 90596268I8, Email chaoran.yi@yahoo.com

Received: December 0I, 2016 | Published: December 14, 2016

\section{Introduction}

Mitral valve prolapse (MVP) is defined as posterior displacement of all or part of MV leaflet(s) beyond MV annulus at least $2 \mathrm{~mm}$ into the left atrium (LA) during systole, with or without mitral leaflet thickening, but prolapsed leaflet(s) pointing towards apex in systole. ${ }^{1}$ Mitral regurgitation (MR) is a common complication of MVP. Moreover, MVP with flail leaflet(s) is a severe form of MVP without systolic coaptation. Compared to simple MVP, flail leaflet tip(s) point towards LA and away from apex in systole, typically associated with torn chordae or ruptured papillary muscle., ${ }^{2,3}$ Flail leaflet is commonly seen with acute and severe MR.

Echocardiography plays vital important role in identifying pathology of MVP with flail leaflet, assessment of MR severity and hemodynamic consequence, and planning MV surgery. I report a case of a symptomatic patient in whom both transthoracic (TTE) and transesophageal (TEE) echocardiography presented an eccentric anteriorly directed severe MR jet as a result of MVP with a flail posterior leaflet due to torn chordae.

\section{Case description}

A 70-year-old female patient presented with shortness of breath (SOB), progressive dyspnea and chest discomfort for two weeks. She has hypertension with medication treatment, without past history of congenital heart disease, coronary artery disease (CAD), arrhythmia, lung disease and diabetes. A typical grade $3 / 6$ panststolic murmur was heard at the apex with radiation to the axillae. The complete blood count, electrolytes, chest X ray and ECG tests were within normal limits. TTE demonstrated severe MVP with flail posterior leaflet, which was consistent with an eccentric anteriorly directed severe MR jet. TEE confirmed TTE echo findings and revealed localized P2 segment defect involved in this pathology. Afterwards, she took Lasix (Diuretics) to control her symptoms, together with Ramipril (ACEI), followed by an operation of minimally invasive MV repair.

\section{TTE and TEE sonographic findings}

i. The hallmark of echocardiogram showed:

ii. Normal size of LV with hyperdynamic systolic function (EF 83\%, Simpsons's BP). iii. Moderately increased LA volume.

iv. Thickened MV leaflets with partial flail of $\mathrm{P} 2$ segment of posterior leaflet, and torn chordae (Figure 1).

v. Eccentric anteriorly directed severe MR jet area over $50 \%$ (Figure 2) with MR ERO $0.47 \mathrm{~cm} 2$ (Figure 3) and Vena Contracta $0.76 \mathrm{~cm}$ (Figure 4), which is consistent with posterior flail leaflet of MVP.

vi. Systolic reverse blood flow in pulmonary vein (Figure 5) and dominant $\mathrm{E}$ wave in MV inflow with elevated LA pressure and high LV diastolic pressure (Figure 6).

vii. Mild pulmonary hypertension (Figure 7).

\section{Diagnosis of MVP with flail posterior leaflet and grading of MR severity}

Physical examination and 2-dimensional (2D) echocardiography are the diagnostic gold standards for MVP. ${ }^{4}$ According to echo findings I listed above, together with the patient's symptoms (SOB and chest discomfort) and pansystolic murmur heard at the apex, the patient was diagnosed as severe MVP with flail posterior leaflet. Echo criteria of flail leaflet includes leaflet tip pointing to LA without systolic coaptation (Figure 1,2,4), torn chordae, double contour (parallel sign) between flail and normal leaflets (Figure 4), and eccentric MR jet direction opposite to the location of the flail leaflet (Figure 2 and 4). ${ }^{3,4}$ Doppler echocardiography is essential in determining the severity of MR. According to ASE guideline, criteria for severe MR includes a vena contracta width $\geq 0.7 \mathrm{~cm}$, large central mitral regurgitation jet (area $>40 \%$ of left atrium), pulmonary vein reversal, effective regurgitant orifice $>40 \mathrm{~mm}^{2}$ and others. ${ }^{3,5}$ The echo findings and Doppler measurements strongly support a severe MR in the patient (Table 1).

Table I Grading of MR severity base on ASE guideline for severe MR

\begin{tabular}{|c|c|c|}
\hline & ASE Guideline 5 & The Case Study \\
\hline \multirow[t]{2}{*}{$\begin{array}{l}\text { Specific signs } \\
\text { of severity }\end{array}$} & $\begin{array}{l}\text { Vena contracta width } \\
\geq 0.7 \mathrm{~cm}\end{array}$ & $0.76 \mathrm{~cm}(\mathrm{TEE})$ \\
\hline & $\begin{array}{l}\text { Large central MR jet (area) } \\
40 \% \text { of LA) or variable size } \\
\text { wall-impinging jet swirling } \\
\text { in LA }\end{array}$ & $\begin{array}{l}\text { Eccentric wall impinging } \\
\text { jet swirling in over } 50 \% \text { of } \\
\text { LA (TTE) }\end{array}$ \\
\hline
\end{tabular}


Table Continued...

\begin{tabular}{|c|c|c|}
\hline & ASE Guideline 5 & The Case Study \\
\hline & $\begin{array}{l}\text { Large flow convergence } \\
0.9 \mathrm{~cm} \text { at Nyquist of } 40 \mathrm{~cm} / \mathrm{s}\end{array}$ & $\begin{array}{l}1.1 \mathrm{~cm} \text { at } 31.9 \mathrm{~cm} / \mathrm{s} \\
\text { converted to } 0.98 \mathrm{~cm} \text { at } \\
40 \mathrm{~cm} / \mathrm{s}\end{array}$ \\
\hline & $\begin{array}{l}\text { Systolic reversal in } \\
\text { pulmonary veins }\end{array}$ & Positive \\
\hline & $\begin{array}{l}\text { Flail MV leaflet with } \\
\text { ruptured chordae or } \\
\text { papillary muscle }\end{array}$ & $\begin{array}{l}\text { Partial flail leaflet of } \\
\text { posterior MV leaflet (TTE } \\
\text { and TEE) with P2 segment } \\
\text { (TEE) }\end{array}$ \\
\hline \multirow[t]{2}{*}{$\begin{array}{l}\text { Supportive } \\
\text { signs }\end{array}$} & $\begin{array}{l}\text { Dense, triangular CW } \\
\text { Doppler MR jet }\end{array}$ & $\begin{array}{l}\text { Dense, early peaking, } \\
\text { asymmetrical MR jet }\end{array}$ \\
\hline & $\begin{array}{l}\text { E-wave dominant mitral } \\
\text { inflow }(E>1.2 \mathrm{~m} / \mathrm{s}) \text {, enlarged } \\
\mathrm{LV} \text { and } \mathrm{LA}\end{array}$ & $\begin{array}{l}\text { E-wave } 1.27 \mathrm{~m} / \mathrm{s} \text {, normal } \\
\text { LV size with increased LV } \\
\text { diastolic pressure, and } \\
\text { moderately enlarged LA } \\
\text { volume with increased LA } \\
\text { pressure }\end{array}$ \\
\hline \multirow[t]{2}{*}{$\begin{array}{l}\text { Quantitative } \\
\text { parameters }\end{array}$} & $\begin{array}{l}\text { Vena contracta width } \\
\geq 0.7 \mathrm{~cm}\end{array}$ & $0.76 \mathrm{~cm}$ (TEE) \\
\hline & $E R O A \geq 0.4 \mathrm{~cm} 2$ & $0.47 \mathrm{~cm} 2$ \\
\hline
\end{tabular}

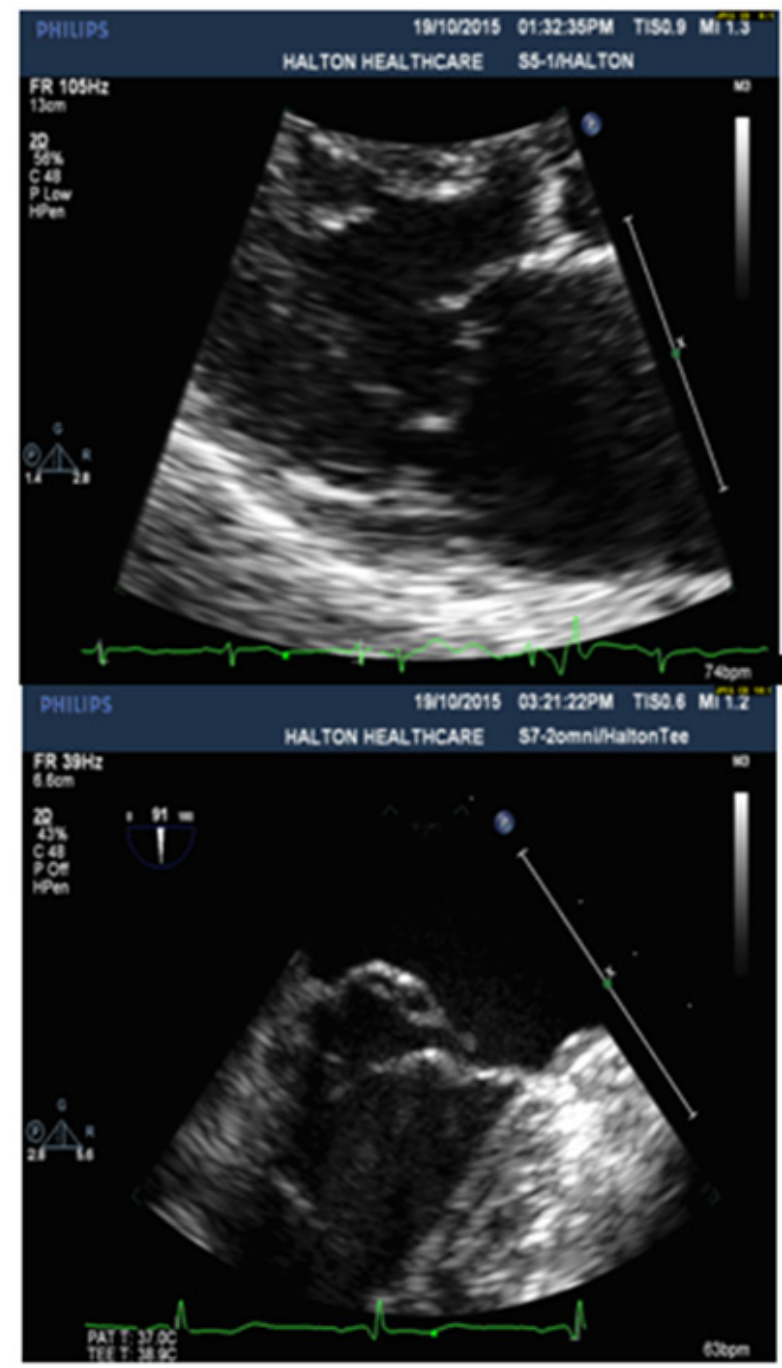

Figure I Severe MVP with Partial Flail Posterior Leaflet presented by parasternal LAX view with TTE (Left) and sagittal view at 9l $\circ$ with TEE (P2 segment) (Right).

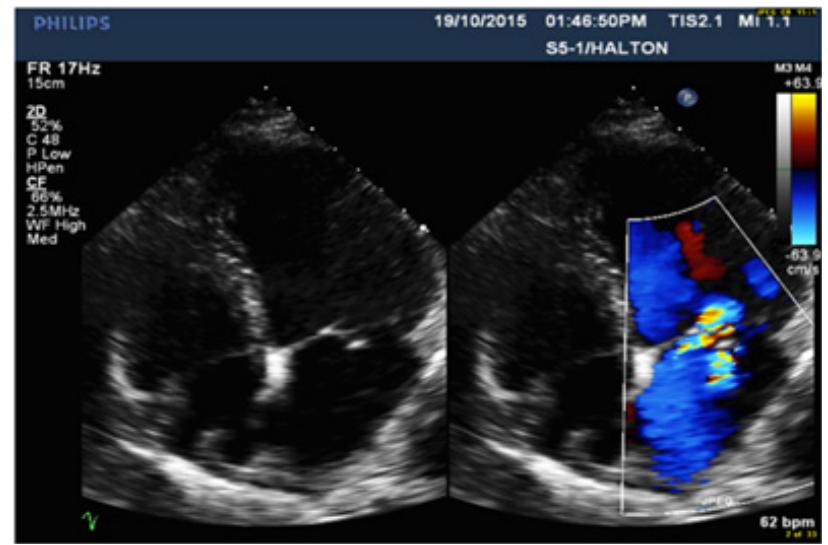

Figure 2 Eccentric wall impinging Jet Area over 50\% is anteriorly directed, consistent with flail posterior MV leaflet.

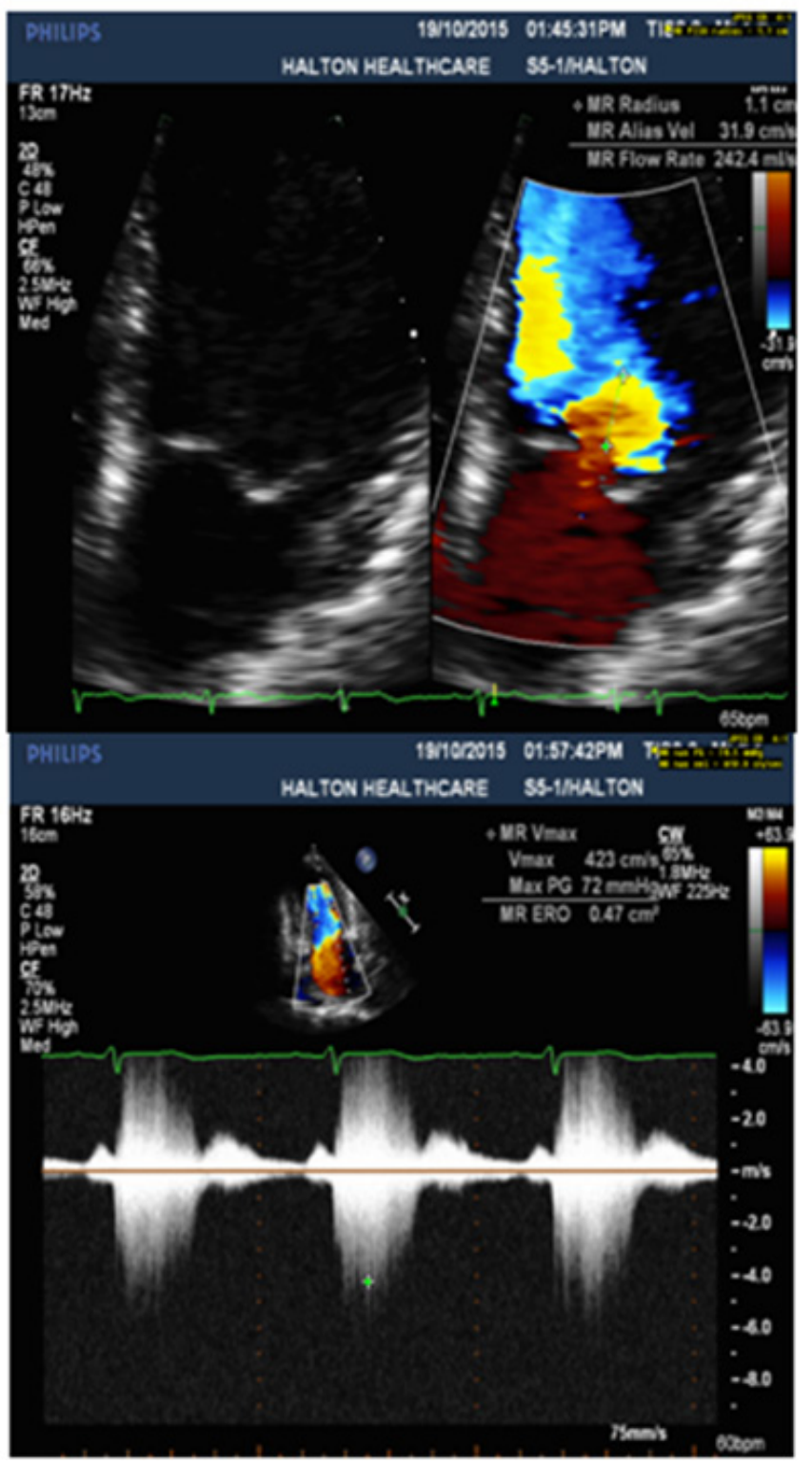

Figure 3 PISA: large flow convergence radius $1 . I \mathrm{~cm}($ Left) with MR ERO 0.47 $\mathrm{cm} 2$ and dense, early peaking and triangular CW Doppler MR jet(Right). 


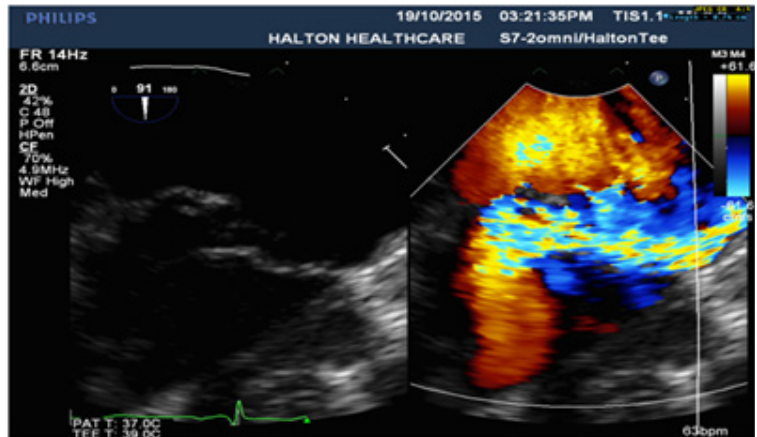

Figure 4 Anteriorly directed eccentric MR jet (TEE) due to P2 segment defect of flail posterior leaflet with the vena contracta $(0.76 \mathrm{~cm})$, consistent with severe regurgitation.

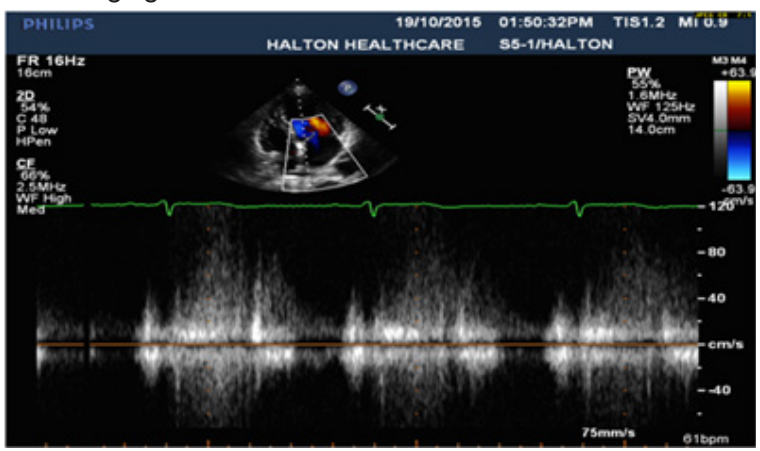

Figure $\mathbf{5}$ Systolic reversal blood flow in pulmonary vein Doppler.

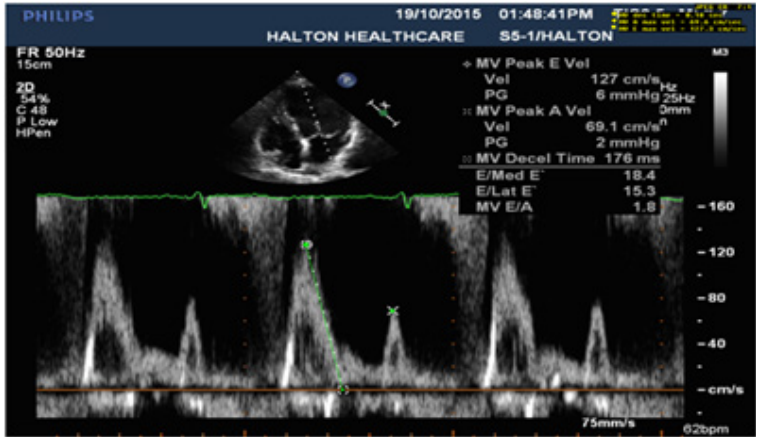

Figure 6 E- wave dominant mitral inflow $(E>1.2 \mathrm{~m} / \mathrm{s})$ with signs of elevated LA and high LV diastolic pressure ( $\mathrm{L}$ wave).

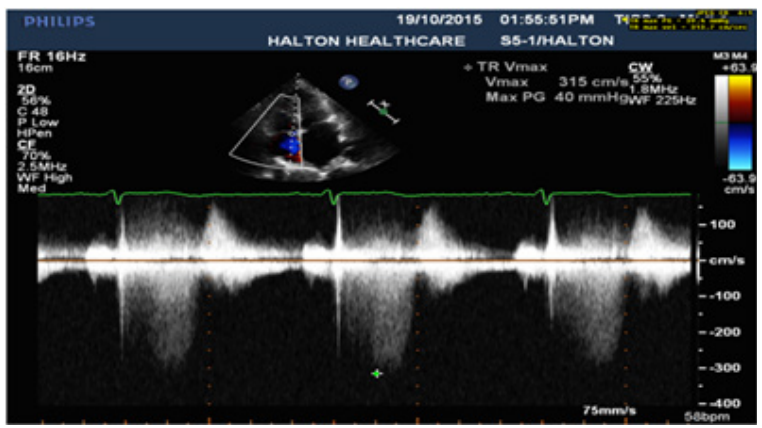

Figure 7 Tricuspid regurgitation: Mildly increased right ventricular systolic pressure, $43 \mathrm{mmHg}$.

\section{Discussion}

\section{Prevalence and Etiology}

The prevalence of MVP is estimated at 2-3\%, while MVP with flail leaflet affects more frequently the posterior leaflet ( $70 \%$ of cases) and is the most common cause of severe non-ischemic MR in the USA. ${ }^{1}$ According to clinical studies and medical research, a couple of causes have been identified as etiology of MVP, including degenerative (myxomatous) valve, fibroelastic deficiency, acute rheumatic valve disease, Marfan's syndrome, bacterial endocarditis, papillary muscle rupture and acute ischemia. ${ }^{3}$ Moreover, myxomatous degeneration and fibroelastic deficiency account for most of MVP. The former may present as a genetic disorder while the latter might be caused as an accelerated aging process. Furthermore, flail leaflet(s) of MVP has been found in more than $50 \%$ of myxomatous valves with ruptured chordate. ${ }^{3,4}$

\section{Pathophysiology}

In this case, torn chordae leading to partial flail posterior MV leaflet caused sub-acute significant MR, followed by significant increased LA pressure due to sudden increased LA volume overload from severe MR in systole. Simultaneously, hyperdynamic LV systolic function maintains forward flow due to increased preload according to Frank Startling Law. ${ }^{6}$ Consequently, the patient demonstrated SOB, progressive dyspnea and chest discomfort due to pulmonary hypertension from increased LA pressure.

The time of significant MR can be acute or chronic stage. However, significant difference of hemodynamic consequences exist between acute stage MR and chronic stage MR. In the stage of acute MR, usually we can see a normal size of LV with hyperdynamic systolic function and high LA pressure, accompanied by pulmonary hypertension while a huge dilated LV size (eccentric hypertrophy) and dilated LA volume size with normal or mildly increased LA pressure can be commonly seen in the chronic stage. ${ }^{6}$ In this case, I consider the patient had a sub-acute stage of significant MR because of normal size of LV with hyperdynamic systolic function $(83 \%)$, moderately increased LA volume size due to previous possible mild MR from simple MVP, together with significant MR from flail leaflet, symptoms over two weeks and evidence of flail posterior leaflet in the new echo findings.

\section{Differential diagnosis}

When MVP with flail leaflet was diagnosed by TTE, next step should consider which scallop segment can be involved in the defect. TEE is effective in identifying prolapsing segments with multiple plans. Anatomically, the posterior and anterior leaflets of the mitral valve each may be divided into three sections. Carpentier's widely recognized nomenclature describes three posterior leaflet scallops-the lateral (P1), middle (P2), and medial (P3)-and three anterior segments (A1), (A2) and (A3). ${ }^{2,3}$ According to clinical studies, P2 is the most common site of localized prolapse and flail due to myxomatous degeneration and fibroelastic dysplasia. ${ }^{2}$ In this study, localized P2 segment defect was identified by sagittal view (long axis) of TEE.

Additionally, differential diagnosis should also consider various causes of MR, including mitral annulus calcification, MV perforation due to infective endocarditis, functional MR (dilated cardiomyopathy), rheumatic MR with typical sign of commissural fusion, and ischemic MR with the history of myocardial infarction and CAD. ${ }^{5,6}$

\section{Technical quality and limitations}

Most of images presented good quality and accurate measurements in this study. However, M-Mode on MV tips didn't show a typical holosystolic "sagging" of flail posterior leaflet in systole. In my opinion, a couple of limitations were related to this issue including artifacts from thickened leaflet, inappropriate perpendicular angle 
between cursor and leaflet tips, irregular movement of partial flail leaflet and patient's symptom of SOB .

\section{Treatment and Follow Up}

The medical treatment of MR entails afterload reduction, diuresis, and improve symptoms of heart failure. ${ }^{2}$ Valve surgery is clearly indicated for patients with symptomatic severe MR. ${ }^{1,2}$ MV repair offers several advantages over MV replacement, including lower operative mortality, better long term survival, lower risk of thromboembolic events or significant hemorrhage, and improved post-operative LV function. ${ }^{1}$ Regular follow up and clinical assessment is needed every 6 months and an echocardiogram would be performed every lyear.

\section{Conclusion}

MVP with flail leaflet is a common cause of acute or sub-acute severe MR. Echocardiogram plays vital important role in diagnosis of MVP with flail leaflet and grading of MR severity. TEE can accurately identify the underlying mechanism of MR, involved scallop segment of flail leaflet and for planning MV surgery. MV repair is the preferred treatment for symptomatic prolapse with flail leaflet and severe MR.

\section{Acknowledgments}

None.

\section{Conflicts of interest}

Author declares there is no conflicts opf interest.

\section{Funding}

None.

\section{References}

1. Hayek E, Gring $\mathrm{CN}$ and Griffin BP. Mitral valve prolapse. Lancet. 2005;365(9458):507-518.

2. Guy TS, Hill AC. Mitral valve prolapse. Annu Rev Med. 2012;63:277-292.

3. Shah PM. Current concepts in mitral valve prolapse - diagnosis and management. J Cardiol. 2010;56(2):125-133.

4. Delling FN, Vasan RS. Epidemiology and pathophysiology of mitral valve prolapse: new insights into disease progression, genetics and molecular basis. Circulation. 2014;129(21):2158-2170.

5. Zoghbi WA, Enriquez-Sarano M, Foster E, et al. Recommendations for evaluation of the severity of native valvular regurgitation with twodimensional and Doppler echocardiography. $J$ Am Soc Echocardiogr. 2003;16(7):777-802

6. Otto CM. Valvular Regurgitation. Otto CM, Textbook of Clinical Echocardiography. (5th edn), Saunders Elsevier, Philadelphia, USA. 2013;305-341. 